\title{
Interfacial Analysis in AZ31B Magnesium Alloy Reinforced with Multiwalled Carbon Nanotubes
}

\author{
Cesar A. Isaza $\mathrm{M}^{1}$, Y. Cardona-Maya ${ }^{2}$, J. M. Herrera-Ramirez ${ }^{3}$, C. Carreno-Gallardo ${ }^{3}$ and J. M. Meza ${ }^{4}$. \\ 1. Institución Universitaria Pascual Bravo, Facultad de Ingeniería, Grupo GIIEN, A. A. 6564, campus \\ Robledo, Medellín, Colombia. \\ 2. Departamento de Ciencias Básicas, Universidad Católica Luis Amigó, Transversal 51A \#67B 90, \\ Medellín, Colombia. \\ 3. Centro de Investigación en Materiales Avanzados (CIMAV), Laboratorio Nacional de \\ Nanotecnología, Miguel de Cervantes 120, 31136 Chihuahua, Chih., México. \\ 4. Universidad Nacional de Colombia, Facultad de Minas, Departamento de Materiales y Minerales $\mathrm{Cl}$ \\ 75 No 79A 51, 050032. Colombia. \\ * Corresponding author: c.isaza2059@pascualbravo.edu.co
}

Metal matrix composites (MMCs) have applications in many industries. In particular, magnesium alloys and composites are interesting for both scientific research and commercial applications because of their low density [1]. The multiwalled carbon nanotubes (MWCNTs) have been considered as an ideal reinforcing material to improve the mechanical behavior of alloys due to their mechanical properties [2]. Because of this, researchers around the world have been studying several processes and methodologies for developing new light materials reinforced with carbon nanotubes [3]. In this way, a novel technique, called sandwich technique, was reported to be successful in producing MMCs, such as AZ31B magnesium alloy reinforced with MWCNTs, with good mechanical properties [4]. The mechanical properties of the light composite depend on the chemistry, atomic structure and the bonding at the interface, since this is the region where the mechanical load is transferred from the light matrix to the reinforcement. In this study, the interface between AZ31B magnesium alloy and MWCNTs were studied by microscopy techniques, in order to understand the good mechanical properties reported in our previous work [5].

Figure 1 shows TEM and HRTEM images of the raw MWCNTs, which reveal outer diameters ranging from 10 to $40 \mathrm{~nm}$, inner diameters between 10 and $20 \mathrm{~nm}$ and lengths between 30 and $50 \mu \mathrm{m}$. Some imperfections, including variable number of carbon layers and amorphous carbon defects, can be identified. Figure 2 presents FESEM images of the composite and a close-up of the interface between the magnesium layers, i.e. the reinforced zones or the MWCNTs-rich zones. In general, this image shows a good dispersion of the MWCNTs; in addition, MWCNTs clusters were not observed, which allows promoting a good load transference between the magnesium matrix and MWCNTs, which in turn produces good mechanical properties. For the interface characterization between magnesium and MWCNTs, TEM and HRTEM techniques were used. Dislocations stacking and coherence or semicoherence structure between magnesium matrix and MWCNTs were found, as shown in Figures 3 and 4.

\section{References:}

[1] Kulekci, M.K., The Intern. Journal of Advanced Manuf. Technology 39(9-10) (2008), p. 851.

[2] Treacy, M.J., T. Ebbesen, and J. Gibson, Nature 381(6584)(1996), p. 678.

[3] Tjong, S.C., Materials Science and Engineering: R: Reports 74(10) (2013), p. 281.

[4] C. Isaza, G. Sierra, J. Meza, Journal of Manufacturing Science and Engineering 138 (2016) 024501.

[5] Cesar A. Isaza Merino et al., Journal of Alloys and Compounds 707 (2017): 257-263. 


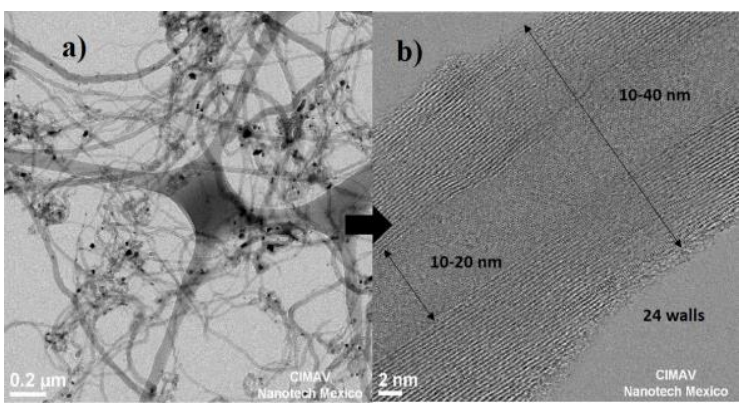

Figure 1. a) TEM and b) HRTEM images of multiwalled carbon nanotubes.

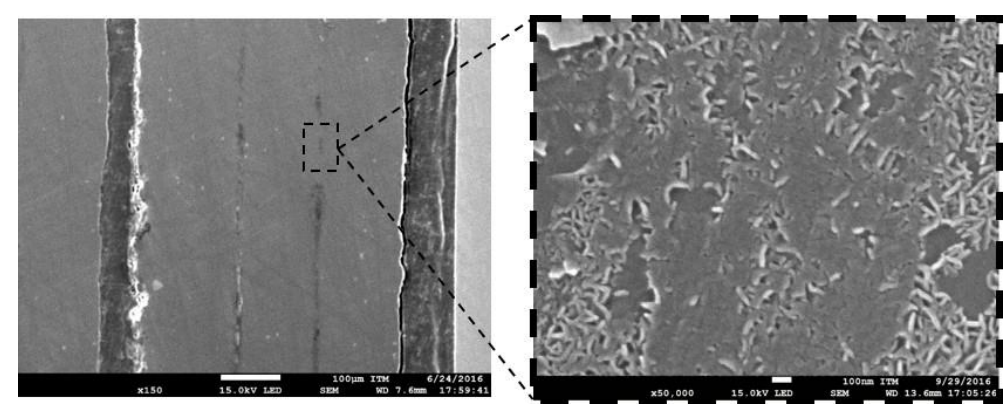

Figure 2. FESEM images of AZ31B magnesium alloy reinforced with MWCNTs.

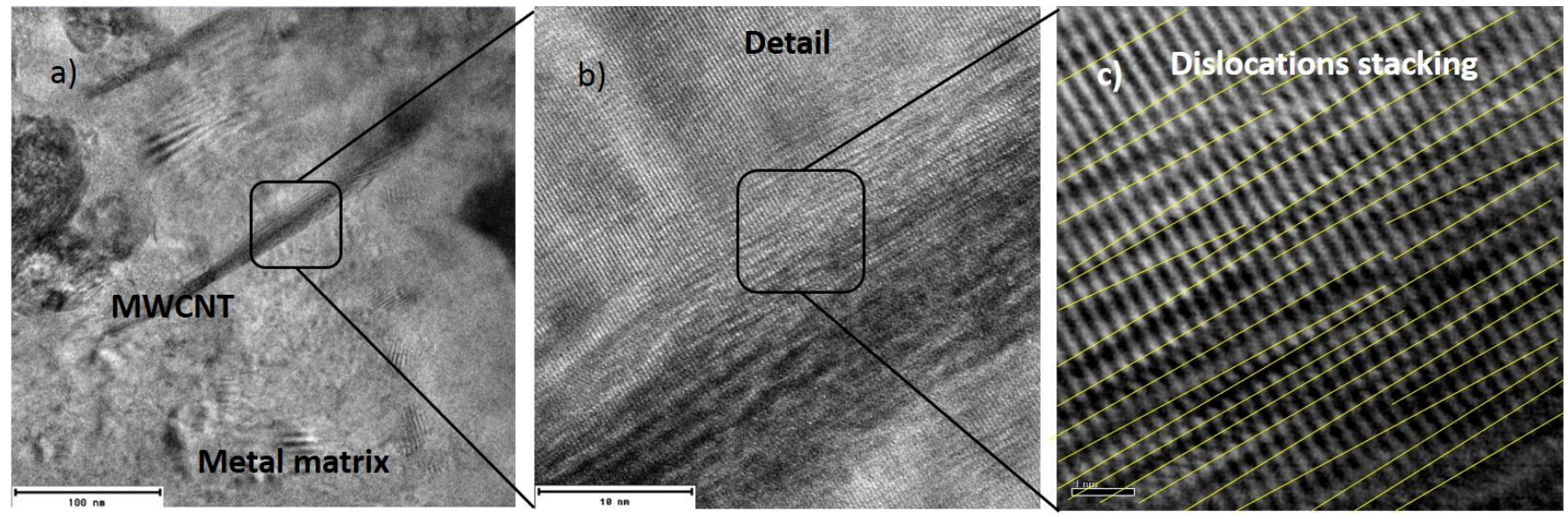

Figure 3. HRTEM images for the composite reinforced with MWCNTs; a dislocation stacking can be seen.

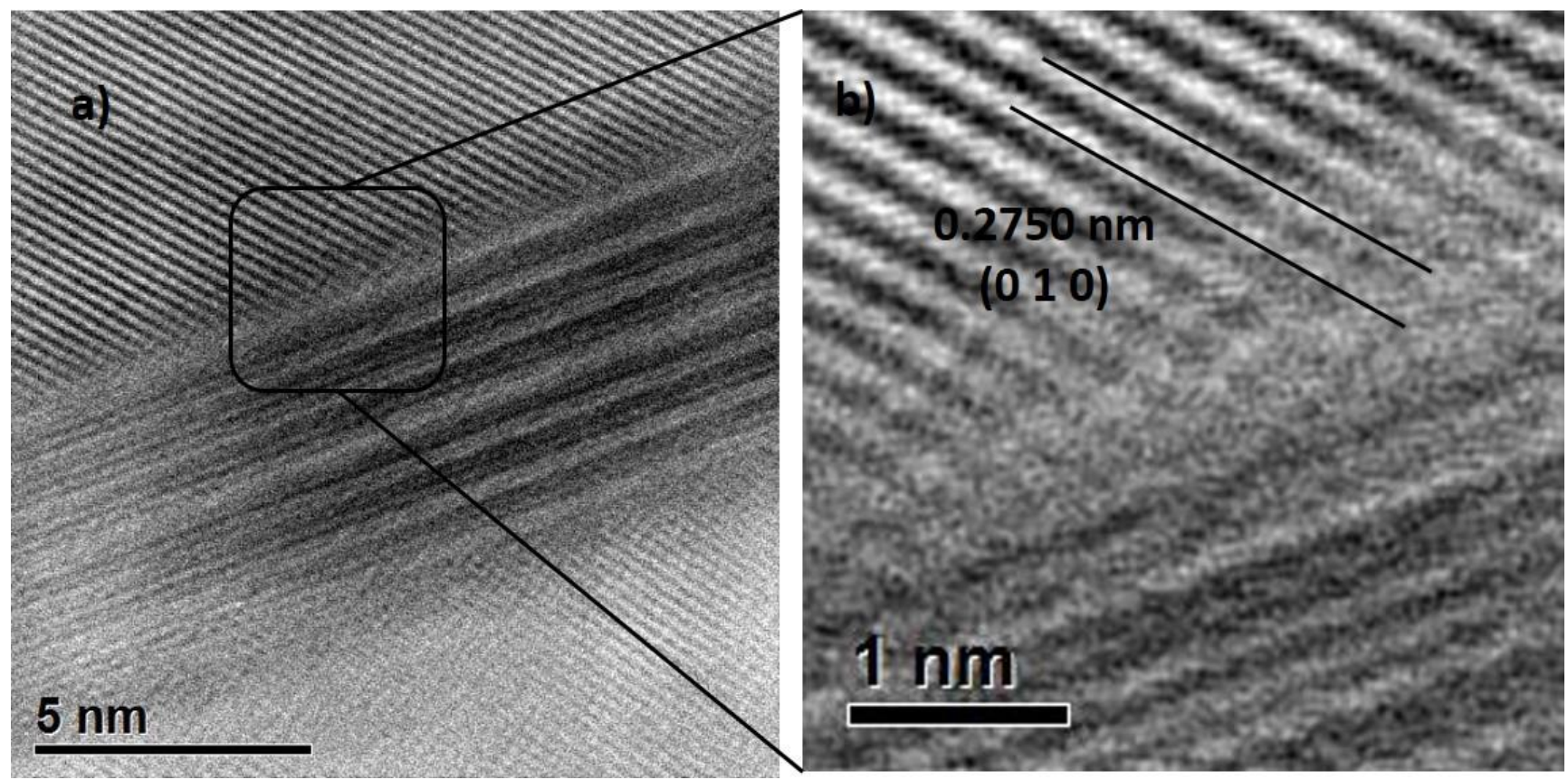

Figure 4. HRTEM images for the composite reinforced MWCNTs; coherence between phases can be seen. 